\title{
TRAUMA-INFORMED SELF-CARE FOR CHILD AND YOUTH CARE WORKER
}

\author{
Vaughan Bowie \\ University of Western Sydney
}

This article presents a framework for understanding, developing, and applying a trauma-informed approach to staff, team, and organizational self-care in youth serving organizations. The article draws on current research in the areas of Adverse Childhood Experiences (ACE), Trauma-Informed Care (TIC), Trauma Stewardship, resilience, and The Sanctuary Model. This approach will help child and youth care workers to develop a long lasting, effective trauma-informed self, team, and organizational care plan. The first article focuses on worker traumainformed self-care, and the second on team and organizational approaches.

Since there are limits as to what can be comprehensively covered in a short article, I will supply an extensive list of key references and resources relevant to self, team, and organizational care. Many of these are directly downloadable from the Internet. Thus, I do not attempt to answer all the issues but encourage readers to explore the various resources and ideas and apply them where relevant to the readers, their team, and organizations.

\section{The Adverse Childhood Experiences (ACE) Study and Its Implications for Child and Youth Care Workers}

The ACE study was undertaken in the United States in the late 1990s (Felitti et al., 1998). The United States Center for Disease Control and Kaiser Permanente, a health insurance company, collaborated in the 10 -year study involving 17,337 adults, focusing on the effects of adverse childhood experiences over the participants' life spans.

The questions in this survey related to a number of adverse childhood experiences:

- physical, emotional, or sexual abuse

- neglect or abandonment

- loss of parent due to divorce or death

- alcoholism or drug addiction in the family

- family violence

- lack of food and basic needs

- family member in prison

- family member with mental illness. 
The survey respondents were largely white, middle class, educated people from professional and skilled trades backgrounds. The study found that around one third of those surveyed had no adverse childhood experiences, with $16 \%$ having four or more adverse childhood experiences, and 9\% having five or more ACE. This and other research have shown that the more adverse childhood experiences encountered by an individual the higher the correlation with later poor health and risky behaviors (Cooper, 2007; Hodas, 2012a).

So what were the ACE effects on this relatively well-off group? Felitti et al. (1998) note that the impacts of such adverse childhood experiences are neurological, biological, psychological, and social. These can include neurobiological changes in the structure and functioning of the brain neurobiology and related cognitive, emotion$\mathrm{al}$, and social impairments at various stages of development. The earlier in life the trauma occurred, the more pervasive these changes can be. These impairments can lead to health-related risk behaviors in order to cope. Such behaviors may include eating disorders, drug and alcohol abuse, inappropriate sexual behavior, self-harm, and violence, creating severe and persistent behavioral health and social problems including obesity, heart disease, and smoking, which can lead to early death.

Compared to this first relatively well-off group, what is the level of ACE in the at-risk youth populations that child and youth care workers deal with? One study found that in a school in Washington state serving lower income students, 10 of 30 children had an ACE score of four or more (Anda, 2012). The prevalence of data indicates that the majority of adults and children in mental health treatment settings, and welfare, correctional, and addiction services have major trauma histories. Cooper (2007) noted that in the United States "Many children and youth experience trauma. Depending on their circumstance, $25-90 \%$ of children and youth experience events that leave them traumatized" (p. 1). These include up to $50 \%$ of children and young people in the child welfare system, from $60-90 \%$ of youth in juvenile justice settings, and 59-91\% of children and adolescents in the community mental health system (Cusack et al., 2003; Cooper, 2007; Hodas, 2006; 2012a; Michelfelder \& Swoboda, 2012).

\section{Obvious Causes of Trauma}

Experiencing such trauma can lead to immediate responses in children and youth, such as being hypervigilant to danger, the narrowing of visual and mental focus resulting in restlessness. There may also be the opposite reactions of feelings of helplessness, lethargy, and freezing, and also denial and dissociation (Hudson, n.d.; Levine, 2008; Michelfelder \& Swoboda, 2012). Other less immediate responses can include disturbed sleep patterns, nightmares, flashbacks, or "daymares." Other responses include being hypersensitive to noise, light, and smell, being easily startled or emotionally over-reactive, and having mood swings. There may also be a general inability to deal with stress and an overwhelming sense of shame and lack of self-worth (Hodas, 2012a). 
Longer-term responses to adverse childhood experiences can lead to panic attacks, phobias, and avoidance of certain situations. There may also be the inability to think and concentrate easily, memory loss of the incident, or a sense of being spaced out. Some adolescent trauma survivors may display over time drug and alcohol addictions, uncharacteristic sexual behavior, or the seeking of stimulating and dangerous situations. At the same time, trauma survivors may have a well-founded perception of impending death and display self-harm or explosive aggression. Sometimes as a result of attachment disruption such people show an inability to love, nurture, or form positive relationships.

Trauma responses can be stable - that is, continually present-or unstable, reemerging when triggered by certain situations. They can also remain hidden for long periods of time and then suddenly appear at key life transitions or periods of crisis (Levine, 2008). Terr (1991) noted two sorts of trauma: type one, which involves a single incident, and type two, which involves multiple traumas over an extended period. However, in passing it must be noted that some trauma survivors acknowledge that often from trauma arises personal growth and resilience or hardiness, which is the so-called salutogenic effect (Conchar \& Repper, 2014).

\section{Trauma Mastery}

One of the most intriguing and sometimes baffling responses by service users to adverse childhood experiences and other life experiences is trauma mastery. For many of those who survive trauma, one of the most difficult and damaging aspects of such experiences is the perceived total lack of control. Van Dernoot Lipsky and Burk (2009) noted "What humans often do to reconcile the lack of control is to create and recreate situations as similar to the traumatic incident as possible. We seek to turn a traumatic situation in which we once felt powerless into a new situation where we feel competent and in charge." Furthermore, "We tell ourselves that this time there will be a different outcome. Or so we hope... We act reflexively, attempting to salvage some sense of control" (p. 156).

However, in an attempt to gain trauma mastery over their circumstances, service users may fail once more to regain power and control and further increase their sense of powerlessness and trauma reactions. At the most extreme responses, unconscious attempts at trauma mastery may trigger returning to dangerous settings and the possibility of greater physical and psychological damage. Some service users may continue to live or remain in dangerous situations or attempt to provoke negative behaviors from others against themselves. However, it should be noted that service users may also actively seek to remove themselves from such trauma-invoking situations and not try to gain trauma mastery at all. The avoidance of trauma-related cues and memories is one of the four symptom clusters that comprise posttraumatic stress disorder.

In light of the above information, those in the system have a history of traumatic stress, and we need exercise universal precautions by creating systems of care 
that are trauma-informed (Hodas, 2006; 2012b). So increasingly trauma-informed services are being built around this concept of universal precautions. In some rare instances this may not be the case, but it seems wise to take this approach until proven wrong.

\section{Adverse Childhood Experiences Histories in Child and Youth Care Workers}

How different are the adverse childhood experiences of child and youth care workers from those they serve? The current limited research on the trauma history of human service workers (Black, 2008; Maunder et al., 2010; Michalopoulos \& Aparicio, 2012; Conchar \& Repper, 2014) has found a wide range of adverse childhood experiences from a low of around $30 \%$ to a high of $76 \%$ (one in three of the middle-class group had no adverse childhood experiences history). Those surveyed included youth workers, counselors, faith-based workers, psychologists, social workers, health staff, and lawyers. Anecdotally, many of these practitioners have mentioned a trauma event or events as being the major motivation for wanting to enter child and youth care work or other human services work.

In light of the above research around staff ACE, many service providers have similar backgrounds to those they care for. Bloom and Farragher (2011) commented, "The bottom line is that there is no clear dividing line between ' $u s^{\prime}$ and 'them,' between the people who need help and the people who offer help. Frequently, the helpers are themselves wounded warriors of a different sort" (See also Auw, 1991, 1999, p. 68).

Because of these relatively high levels of adverse childhood experiences in human services workers, including child and youth care specialists, it is vital to take into account the trauma histories of staff to ensure their well-being. Because of the young people they serve, there must be an awareness by employing organizations of the strong possibility that many employees themselves have trauma histories and will need additional supports. Issues of employment law and employee confidentiality may hamper the efforts of organizations to address these issues unless innovative approaches for Trauma Informed Teamwork (Bowen, 2015) are used for the supporting and training of child and youth care workers. This topic will be covered more fully in the next article.

Part of this approach is to provide the opportunity for child and youth care workers to examine how their own childhood and later life experiences may affect the way they deal with stress and trauma in themselves and the people they serve (Conchar \& Repper, 2014). This is not always easy, as Reese and Loanne (2012) point out, "Our early years are fundamental in our development. Paying attention to the different critical incidents of these early years is in many ways hard ... work, but it can also be very freeing and healing ..." (p. 83). Arledge and Wolfson (2001) refer to such staff as "clinician survivors" (p. 92). An important part of such early childhood experiences for current staff can be their types of early attachment to their caregivers or parents. Such attachments can affect staff members' later expe- 
riences of how they care for themselves and others (Scazzero, 2006; Bennett, 2008; Simmonds et al., 2009).

Such an examination can be encouraged within the organization in a number of supportive ways, including external mentoring, supervision, and therapy, either done individually or in small groups.

\section{Aspects of Four Lenses That May Be Key in the Way One Cares for Oneself and Others}

When child and youth care workers have a significant exposure to ACE, they can be more affected by the behaviors of the youth and children they serve than other staff who have no, or fewer, ACE. Even though the staff may not be diagnosed with PTSD, their behavior may reflect some of the hallmarks of this stress-related disorder. Vicarious traumatization is a negative change that occurs within those helping others who are trauma survivors (Saakvitne \& Pearlman, 1996). Vicarious trauma is about having too much - too much trauma, threat, and feeling overwhelmed and dehumanized. Reese and Loanne (2012) suggest that there are four particular lenses that can help identify important aspects of one's early formation:

(a) one's family history,

(b) one's historical and cultural context,

(c) one's social base, and

(d) one's spiritual background.

Parts of these lenses are the various critical incidents people face during their early and ongoing development. "A critical incident can be any key relationship, event, or circumstance that has had significant influence in shaping your life, whether that incident was positive or negative" (Reese \& Loanne, 2012, p. 96). These early experiences may then become the default position in the way people treat themselves and those they care for. Because of these experiences, people "Tend to favor certain areas of self-care and neglect others, depending upon our personal preferences" (Harris, 2001, p. 260).

It is important then that in pre-service training, ongoing professional development, supervision, and mentoring that these four lenses be gently and respectfully examined on a regular basis. This needs to be done in a way that does not retraumatize the worker or team (Bowie, 2013).

\section{Our Current Life and Work Experiences}

A fifth lens should be added, that of one's current multiple relationships at a personal, work, and client level. Stamm's (2010) Professional Quality of Life Scale (ProQOL) is a useful tool to identify the impact of these various areas. Such relationships can induce a number of experiences, both positive and negative, that impact the life and work of youth and child care specialists. Let us now have a brief 
look at compassion satisfaction, compassion fatigue, burnout, posttraumatic stress disorder (PTSD), and vicarious trauma.

There is much information and many resources available on these issues, but a trauma-informed perspective is lacking on them (Child Trauma Academy, n.d.; Skinner \& Roche, 2005; Bowie, 2008; Pearlman \& McKay, 2008; Volk, 2008; Conrad, 2010; CME Resources, 2011). These show how a trauma-sensitive framework can support and encourage positive experiences and minimize the impact of negative incidents.

\section{Compassion Satisfaction-The "Good Stuff"}

Compassion satisfaction arises from the positive aspects of helping related to providing care for and with others within a supportive team and organization. It is also influenced by the workers' beliefs about themselves and their views on giving and helping.

\section{Compassion Fatigue-The "Bad Stuff"}

Compassion fatigue is the flip side of compassion satisfaction and arises from the negative aspects of helping others. This may arise from unhealthy self-perceptions, stresses of working with colleagues in toxic systems, and work-related trauma. It may be an aspect of burnout, a sense of being overwhelmed and worn out by the helping task and lacking effectiveness.

\section{Burnout}

Burnout is an experience of not having enough-not enough energy, time, resources, or successes.

\section{Posttraumatic Stress Disorder and Vicarious Trauma}

Another aspect of the compassion fatigue experience can be work-related traumatic stress that can have two aspects. The first is a direct primary traumatic experience by a staff member, such as a violent attack or the death of a child. In such extreme situations a staff member could develop PTSD. The second, more common experience is that of secondary trauma experiences called vicarious trauma or secondary traumatic stress. Vicarious traumatization is a negative change that occurs within those helping others who are trauma survivors (Saakvitne \& Pearlman, 1996). Vicarious trauma is about having too much—-too much trauma, threat, and feeling overwhelmed and dehumanized.

Workers can be vulnerable to vicarious trauma if they have had a number of adverse childhood experiences or later adult critical incidents and currently lack personal and professional support (Arledge \& Wolfson, 2001). This can be heightened by an inability to draw professional life and work boundaries and to care for one's self physically, emotionally, and socially. Conrad (2010) noted that vicarious trauma can negatively affect the workers' sense of trust, safety, control, 
self-esteem, and the ability to form close intimate relationships. Vicarious trauma triggers can arise from repeated exposure to the young person's traumatic material and the worker's emotional identification with their service user's experiences. This vicarious trauma can be further heightened by a sense of failing the young person or her or his family and a sense of powerlessness to change the service user's situation or an inability to ask for help (Conrad, 2010).

Question: Have you noticed in yourself or colleagues the impact of vicarious trauma in any of these areas: trust, safety, power, esteem, or intimacy? What have you experienced or observed?

\section{Trauma Mastery in Child and Youth Care Workers}

As part of their compassion fatigue experiences, child and youth care workers may also, like service users, attempt to regain, consciously or unconsciously, trauma mastery by repeating actions and behaviors that have not worked in the past, hoping they will the next time. Such attitudes and behaviors may include the following:

- not taking breaks, feeling indispensible

- constant returning to risky situations

- blaming colleagues or team for failures

- isolating self and forming cliques

- claiming superior skills and knowledge

- not setting professional boundaries

- taking responsibility for things they have no control over

- bullying, harassment

- micromanagement

Other staff may try to avoid any attempt at trauma mastery by physically or emotionally withdrawing from dangerous and previously traumatizing environments.

Question: What examples of trauma mastery and avoidance have you seen in yourself or other workers? 
Staff attempts at trauma mastery are not always recognized and addressed in stress management and self-care workshops and organizational policy and protocols. This often leads such approaches to be ineffective on any long-term basis. Thus, an important part of trauma-aware approaches is helping workers and teams to identify and deal with such destructive dynamics that impact service users, workers, other staff, and the organization.

\section{What is Trauma-Informed Care?}

Through the ACE study and current neurobiological research and practice, a whole new focus on trauma-informed care (TIC) is beginning to permeate child and youth care services (Bath, 2015; Fallot, 2011; Hodas, 2012b; Bowie, 2013; Perry, 2014; SAMHSA, 2014).

Fallot and Harris (2006) define trauma-informed care and services the following way: "Human service systems become trauma-informed by thoroughly incorporating, in all aspects of service delivery, an understanding of the prevalence and impact of trauma and the complex paths to healing and recovery. Trauma-informed services are designed specifically to avoid retraumatizing those who come seeking assistance" (p. 3). Fallot and Harris (2009) noted that "Creating cultures of trauma-informed care approach to organizational change is built on five core values of safety, trustworthiness, choice, collaboration, and empowerment" (p. 3). A later SAMHSA publication (2014) added another core value: respecting cultural, historical, and gender aspects of the service user's experience.

Six core principles can be applied to service users in more detail by asking the following questions:

1. Safety: How can physical and emotional safety be ensured for service users throughout the human services systems?

2. Trustworthiness and Transparency: How can service users' trust in staff and their services be maximized? How can tasks and responsibilities be made clear while maintaining appropriate boundaries?

3. Peer Support: How can an environment conducive to the development of mutual support networks of service users or consumers be provided?

4. Collaboration and Mutuality: How can collaboration and sharing of power between staff, organizations, and service users be maximized?

5. Empowerment, Voice, and Choice: How can service users' empowerment, input, and skill-building be made a key priority? How can real and effective choices for them be ensured?

6. Culture, History, and Gender: How can the cultural, historical, and gender aspects of the service users' experiences be respected and integrated? 
Bath (2015) condensed these six core principles into safety, connections, and coping with inner turbulence. Fallot and Harris (2009) developed a number of organizational self-assessment tools based on these core principles for service users.

When appropriate, these can be used to develop a more trauma-informed care approach:

- Trauma-Informed Care Organizational Self-Assessment for Consumer-Run Recovery Organizations (n.d.)

- Guide to Completing the Agency Self-Assessment (n.d.)

- Trauma-Informed Organizational Self-Assessment for Child Abuse Prevention Agencies (n.d.)

Question: What areas in your organization need to be more trauma-informed?

Fallot and Harris (2009) commented, "If an organization can say that its culture reflects each of these values in each contact, physical setting, relationship, and activity and that this culture is evident in the experiences of staff as well as consumers, then the program's culture is trauma-informed" (p. 3). The TIC approach in many ways gives greater understanding of why some helping approaches work and others do not. A "you're broken and we are going to fix you" approach generally does not work, but rather a "what happened" and "how can we help you feel safe" relational approach is more healing.

The benefits of a TIC approach are that it increases safety for all involved with the organization and it improves relationships through a healthier social environment. The TIC approach creates a safe and hopeful workplace culture that also cares for caregivers and in turn improves their success and job satisfaction, which has a positive impact on quality of service, and helps decrease negative encounters and incidents (Sharp \& Ligenza, 2012).

Many human service systems still do not regularly and comprehensively inquire about the impact of past and current trauma experienced by service users and staff. Without this knowledge, service providers may make mistakes and have wrong assumptions in the way they respond to and support young people. This may lead unintentionally to the revictimization or retraumatization of those they are trying to help.

Thus, it is best to take a universal precautions approach to trauma in service users' lives with service providers' roles being to respectfully and carefully explore their past and current histories of trauma. Also at the same time it is necessary to ensure that service procedures, protocols, processes, and environments all create a safe, trustworthy, and respectful environment so that service users do not see the service as a source of further distress. Such principles also need to be applied to staff. 


\section{Applying TIC Practices to Staff}

A key aspect of applying trauma-informed approaches in staff self-care is that of trauma stewardship. Van Dernoot Lipsky and Burk (2009) defined this as "[t]he whole conversation about how we come to do this work, how we are affected by it, and how we make sense and learn from our experiences" (p. 6). They also commented that this involves developing and maintaining "[a] long-term strategy that enables us to remain whole and helpful to others and our surroundings even amid great challenges. To participate in trauma stewardship is to always remember the privilege and sacredness of being called to help" (p. 6).

Pearlman and McKay (2008) recommended asking on a regular basis some key questions at an individual, team, and organizational level that can start such a conversation: "Why am I doing this work? Do I know what I am doing and why? How do I measure success in my work? What can I control and not control at work? What are the costs and rewards of my work? How am I changing?" (p. 30). Such inquiries help staff and management to gain and keep a perspective on what they are doing, why they are doing it, and how it is affecting them.

TIC is built on certain core values, but in practice, many child and youth serving organizations make safety and well-being of service users a higher priority than staff. Fallot and Harris (2011) noted "[w]e have come to see, in the past several years, that the experiences of staff members are equally important in these domains ... when they experience these factors in their working environment and in their relationships with supervisors, administrators, and colleagues" (p. 31). All these principles are based on creating a sense of safety, security, and predictability for the service users.

Fallot and Harris (2011) recommended that these TIC Core Principles be considered once more but with a focus on the staff. This can be done by asking the following questions:

1. Safety: How can physical and emotional safety for staff throughout our human services systems be ensured?

2. Trustworthiness and Transparency: How can workers' trust in their fellow staff and their services be maximized? How can tasks and responsibilities be made clear while maintaining appropriate self-care boundaries?

3. Peer Support: How can an environment conducive to the development of mutual support networks of staff be provided?

4. Collaboration and Mutuality: How can collaboration and sharing of power between staff, their organizations, and service users be maximized?

5. Empowerment, Voice, and Choice: How can staff's empowerment, input, and skill-building be made a key priority? How can real and effective choices for them be ensured?

6. Culture, Historical, and Gender Issues: How can the cultural, historical, and gender aspects of the service workers' experiences be respected and integrated? 
It is key to a trauma-informed care approach to staff and team well-being that each of these core principles be examined in detail, and procedures and protocols be developed at all levels to help ensure and maintain staff wellness. This can be done by adapting some of the organizational self-assessment tools such as those recommended by Volk (2008) for use with staff. If staff members feel that these principles are present in their organization, they will generally have greater passion and commitment to their work and those they serve. Kahn (2005) and Modlin (2012) consider a safe environment as a supportive, holding environment.

The TIC approach is the concept of universal precautions, based on the assumption that clients coming into such services will have histories of trauma. However, such a presumption is not always applied in the employing, training, and support of staff. This is despite the previously mentioned growing research showing relatively high ACE levels of trauma in human service professionals (Black, 2008; Bowie, 2013).

This lack of focus on staff safety can be seen in many current organizational initiatives and staff workshops on stress management, burnout, and vicarious trauma that keep repeating the same old mantra of "change your attitude and habits, resources, or situation." They, however, do not incorporate up-to-date information on trauma-informed care as it relates to staff and so may make little difference to staff stress and traumatization levels. Similarly, Hodas (2012b) noted that "[a]11 too often, the outcome for staff working in an organizational structure that is not trauma-informed is 'burnout' and staff turnover" (p. 2).

Such self-care training approaches can also give the impression that the total responsibility for self-care lies with the workers themselves and often neglects or downplays the equally important role, negative or positive, of the team and organizational climate. In its negative form such attributions may lead workers to take on roles and responsibilities that are not theirs to take or to feel guilty over things they cannot control. These responses may trigger further worker attempts at trauma mastery and create further hopelessness and retraumatization (van Dernoot Lipsky \& Burk, 2009; Bloom, 2010).

Reynolds (2011) observed that "[b]urnout sounds like we're toys with disposable batteries that are used up. As if we're not doing enough yoga or drinking enough water, and these are important things ... but self-care is not enough to offset the issues of poverty, violence, and basic dignity people struggle with. No one advocating self-care suggests that it will create the necessary practical changes in the daily-lived realities of clients" (p. 29).

Such blaming or strengthening interventions focusing on increasing individual staff members' coping skills are the most common strategy used to deal with staff under stress, partially because it is the easiest and cheapest. However, such approaches often prove the least effective. Maslach et al. (2001) emphasized the importance of addressing organizational factors because these exert the strongest influence on staff stress, burnout, and workplace violence (see also Kahn, 2005; Bowie, 2011; Bloom \& Farragher, 2013). 
So considering trauma-informed care at the individual level, service providers need to be reminded that self-care is not a substitute for comprehensive team and organizational approaches. If it is used as such, often all that is done is rearranging the staff self-care deck chairs on the organizational Titanic. Thus, the issue of team and organizational self-care will be dealt with in more detail in article two (Bloom \& Farragher, 2011, 2013).

\section{Developing a Staff Trauma-Informed Self-Care Plan}

Volk et al. (2008) offered a self-care assessment tool to show how one is doing in the areas of physical, psychological, emotional, spiritual, workplace, and professional self-care. This self-assessment tool helps identify one's current situation against which one can compare one's progress in self-care. The ProQOL online assessment tool (Stamm, 2010) is also a key resource to gauge how one is functioning and what needs to be managed or changed (Bowie, 2008).

Question: How did you do with your self-care assessment? Any surprises or things that need to change?

A good place to start with developing a plan is the self-care ABCs of Awareness, Balance, and Connection (Pearlman \& McKay, 2008; Bowie, 2008; CME Resources, 2011). Such strategies should not be new to child and youth care workers and may be a revision of what service providers are already doing or should be doing.

\section{Awareness}

Service providers bring their past and present to whatever they do. This includes their beliefs, worldviews, prejudices, biases, social support systems, adverse childhood experiences, illnesses, and family, financial, and social contexts. All this happens within the context of the individuals' internal needs such as safety, significance, self-worth, and success. This also occurs within broader work and social relationships, team, and organizational demands, and the wider sociopolitical context (Stamm, 2010).

The Headington Institute (Pearlman \& McKay, 2008) maintain that selfawareness comprises physical, emotional, and relational self-care as well as spiritual self-care. Each should be considered.

Physical self-care involves making time for exercising regularly, having a healthy diet, drinking enough water, using alcohol moderately, and having sufficient sleep. The use of mindfulness and relaxation techniques may also be important, but not everyone finds such passive approaches work for them and prefer more active strategies.

Emotional and relational self-care includes building and nurturing meaningful relationships, not just Facebook friends. It includes not isolating oneself be- 
cause of one's work and not having people to talk with. A key aspect of this is having a support group of friends and colleagues. An effective support group can provide those who can challenge one, give emotional support, supply how-to-doit information, and supervise and mentor one. Many workers don't have such a support group, or if they do, it may be comprised of only a few people. In such a situation the worker needs to find a support group or set about creating one. This is not an easy task if one is already feeling tired and burned out. One should start earlier rather than too late. It is also key to have good supervision and external mentoring, and counseling if needed.

Question: How good is your support group? What needs to be changed or developed?

Having time to stop, reflect, meditate, or journal are important for self-renewal. Also helpful is enjoying creative activities that absorb one's here-and-now attention and that quiet the internal mind's chatter. It is important for workers to monitor and limit the amount of trauma material they are exposed to through the various forms of media, and to cultivate a sense of hope and humor. It is key to understand the impact of stress and trauma on oneself and others (Skinner \& Roach, 2005; Pearlman \& McKay, 2008; Volk et al., 2008; Bloom, 2010).

Spiritual self-care is also an important but often neglected or downplayed aspect of self-care. This includes identifying one's core values and knowing who or what motivates and inspires one. Music, singing, and being in nature can all be part of one's spiritual self-care. Regular times of meditation and prayer within a faith community can be important. Being passionate about these things can be helpful (Auw, 1991; Pearlman \& McKay, 2008; Palmer, 2009). The Maori approach to wellness (Durie, 1992; Bowen, 2014) includes family health, psychological health, physical health, and spiritual health. Spiritual health focuses on the source of hope for a person. Hopelessness is one of the products of having a significant ACE score and can result in child and youth care workers becoming jaded or apathetic.

\section{Balance}

Balance and self-care involve having the right priorities and expectations about staff work and personal life. One needs appropriate limits and balances. Multitasking and working extended hours do not improve workers' effectiveness. Sometimes by doing less, one may achieve more. One should take regular breaks away from one's desk and service users and schedule uninterrupted time to relax and think.

\section{Connection}

Possibly the greatest growing challenge to the balance of work and life is our constant online connection through electronic media such as emails, phones, and 
texts. One is constantly faced with a sense of urgency rather than what is most important. Staff may dread coming back from holidays because there are so many emails to deal with, if they have not already checked them while on a break. One needs to be able to digitally detox and create times of solitude in order to be more resilient and effective. In some extreme cases workers can become addicted to stress (May, 1991; van Dernoot Lipsky \& Burk, 2009).

Connection and self-care involves connecting with friends, peers, and various support groups and communities. Such support enables workers to enhance their social and emotional well-being and improve their skills to perform their required work tasks. Support can come from family, friends, work colleagues, and managers and supervisors (Workforce Development NCETA, 2005). Connecting with an external mentor can help staff look at wider issues relating to their whole lives and not just their work, thus helping them to gain a sense of objectivity.

Question: So what do you now need to add, subtract, or modify in your own TIC self-care plan in the physical, emotional, relational, and spiritual areas?

\section{A Final Note of Caution}

Organizations will face significant roadblocks in supporting self-care plans for staff due to employment laws in some countries which mandate that health and mental health concerns not be included in personnel decisions regarding hiring, promoting, retaining, disciplining, and termination of employment. Working within this framework of supporting staff legally must be a commitment to support them emotionally and psychologically to withstand and ultimately prevail over the trauma that pervades the social service system.

\section{Conclusion}

This article has drawn on current research in the areas of adverse childhood experiences, trauma-informed care and services, and trauma stewardship to help workers develop a long lasting, effective trauma-informed self-care plan. It also has drawn on theory and practice from resilience approaches as well as The Sanctuary model. However, as previously noted, staff self-care plans will ultimately be ineffective if they are not formulated within the broader staff and organizational context.

The second half of this article in a future edition of this journal will examine more closely the organizational context of workplace trauma. This organizational context, including management style, can often be toxic and trauma-provoking within the workplace itself, impacting on managers, staff, and those using the service. However, this does not necessarily always need to be the case if the principles for trauma-informed care and services outlined in the article are modified and implemented at organizational and societal levels. 


\section{References}

Anda, R. (2012). Adverse Childhood Experiences: Connecting a Developmental Lens to the Health of Society. Retrieved from http://www.in.gov/dcs/ files/2Dr_Anda5steps.pdf

Arledge, E., \& Wolfson, R. (2001). Care of the Clinician: New Directions For Mental Health Services, No. 89, 91-98.

Auw, A. (1991). Gentle roads to survival: Making self-healing choices in difficult circumstances. Boulder Creek, CA: Aslan Publishing.

Auw, A. (1999). The gift of wounding: Finding hope and heart in challenging circumstances. Boulder Creek, CA: Aslan Publishing.

Bath, H. (2015). The three pillars of traumawise care: Healing in the other 23 hours. Reclaiming Children and Youth, 23(4), 5-11.

Black, T. G. (2008). Teaching trauma without traumatizing: A pilot study of a graduate counseling psychology cohort. Traumatology, 14, 40-50.

Bennett, S. (2008). Attachment-informed supervision for social work field education. Clinical Social Work Journal (2008), 36, 97-107.

Bloom, S. L., \& Farragher, B. (2011). Destroying sanctuary: The crisis in human service delivery systems. New York, NY: Oxford University Press.

Bloom, S. L., \& Farragher, B. (2013). Restoring sanctuary. New York, NY: Oxford University Press.

Bowen, B. (2015). Trauma informed teamwork: Understanding the dynamics of the trauma histories of caregivers. In P. Callaghan, N. Oud, J. Hakon Bjorngaard, H. Nijman, T. Palmstierna, \& J. Duxbury (Eds.), Proceedings of the 9th European Congress on Violence in Clinical Psychiatry, (pp. 92-95). Amsterdam: Kavanah.

Bowen, B. (2014). Integrating indigenous models of mental health: Preventing and healing the transgenerational transmission of trauma. Presented at the Inaugural Symposium of the Neurosequential Model of Therapeutics, Banff, Alberta, Canada, July 13, 2014.

Bowie, V. (2013). Trauma-informed care. Youth Studies Australia, 32(4). 
Bowie, V. (2011). An emerging awareness of the role organizational culture and management style can play in triggering workplace violence. In M. R. Privitera, (Ed.), Workplace Violence in Mental and General Health Care Settings. Sudbury, MA: Jones and Bartlett Publishers.

Bowie, V. (2008). Youth workers and stress. Youth Studies Australia, 27(2), 36-44.

Child Trauma Academy (n.d.). The cost of caring: Secondary traumatic stress and the impact of working with high-risk children and families. Retrieved from http://www.childtraumaacademy.com/cost_of_caring/index.html

CME Resources. (2011). Vicarious trauma and resilience course \# 6662. Retrieved from https://www.netcegroups.com/750/Course_6662.pdf

Conchar, C., \& Repper, J. (2014). Walking wounded or wounded healer? Does personal experience of mental health problems help or hinder mental health practice? A review of the literature. Mental Health and Social Inclusion, 18(1), $35-44$.

Conrad, D. (2010). Secondary trauma: Understanding its impact and taking steps to protect yourself. JFK Partners Department of Pediatrics, University of Colorado Health Science Center.

Cooper, J. (2007). Facts about trauma for policymakers: Children's mental health. National Center for Children in Poverty.

Cusack, K., Frueh, C., Hiers, T., Suffoletta-Maierle, S., \& Bennett, S. (2003). Trauma within the psychiatric setting: A preliminary empirical report. Administration and Policy in Mental Health, 30(5), 453-460.

Durie, M. (2012). Te Whare Tapa Wha. Retrieved from http://www.careers.govt.nz/ practitioners/career-practice/career-theory-models/te-whare-tapa-wha

Fallot, R. (2011). Trauma-informed care: A values-based context for psychosocial empowerment. Washington, DC: Institute of Medicine Workshop on Preventing Violence against Women and Children Community Connections.

Fallot, R., \& Harris, M. (2011). Culture shock. National Council Magazine, 2, 30-31. 
Fallot, R., \& Harris, M. (2009). Creating cultures of trauma-informed care (CCTIC): A self-assessment and planning protocol. Community Connections. Retrieved from http://www.healthcare.uiowa.edu/icmh/documents/CCTICSelf-AssessmentandPlanningProtocol0709.pdf

Fallot, R., \& Harris, M. (2006). Trauma-informed services: A self-assessment and planning protocol. Community Connections.

Felitti, V., Anda, R., Nordenberg, R., Williamson, D., Spitz, A., Edwards, V., Koss, M., \& Marks, J. (1998). Relationship of childhood abuse and household dysfunction to many of the leading causes of death in adults. The Adverse Childhood Experiences (ACE) Study. American Journal of Preventative Medicine, 14(4), 245-258.

Harris, R. (2001). 20 minute retreats: Revive your spirits in just minutes a day with simple self-led exercises. London: Pan Book.

Hodas, G. (2006). Empowering direct care workers who work with children and youth in residential care. Harrisburg, PA: Office of Mental Health and Substance Abuse Services.

Hodas, G. (2012a). Child Mental Health Matters 4.

Hodas, G. (2012b). Childhood trauma and trauma-informed care, part II, from "trauma-infirmed" to "trauma-informed," Children's Mental Health Matters, 5, 1-6. Retrieved from http://www.parecovery.org/documents/CMHM_Hodas_112012.pdf

Hudson, H. (n.d.). Trauma-informed care. Wisconsin.

Kahn, W. (2005). Holding fast: The struggle to create resilient caregiving organizations. East Sussex, UK: Brunner-Routledge.

Levine, P. (2008). Healing Trauma. Boulder, CO: Sounds True, Inc.

Maslach, C., Schaufeli, W. B., \& Leiter, M. (2001). Job burnout. Annual Review of Psychology, 52, 397-422.

May, G. (1991). Addiction and grace: Love and spirituality in the healing of addictions. New York, NY: HarperCollins. 
Maunder, R., Peladeaub, N., Savagec, D., \& Lanceea, W. (2010). The prevalence of childhood adversity among healthcare workers and its relationship to adult life events, distress and impairment. Child Abuse \& Neglect, 34, 114-123.

Michalopoulos, L., \& Aparicio, E. (2012). Vicarious trauma in social workers: The role of trauma history, social support, and years of experience. Journal of Aggression, Maltreatment \& Trauma, 21(6), 646-664.

Michelfelder, M., \& Swoboda, E. (2012). Trauma 101. Trauma-informed care stakeholders group training subcommittee.

Modlin, H. (2012). Organizational social context as a holding environment in child welfare, mental health and youth corrections settings. Presented at 10th Triennial International Child and Youth Care Conference, Canmore, AB.

Mueser, K. T., Goodman, L. B., Trumbetta, S. L., Rosenberg, S. D., Osher, C., Vidaver, R., Auciello, P., \& Foy, D. W. (1998). Trauma and posttraumatic stress disorder in severe mental illness. Journal of Consulting and Clinical Psychology, 66(3), 493-9.

Palmer, P. (2009). Let your life speak. San Francisco, CA: Jossey-Bass.

Pearlman, L., \& McKay, L. (2008). Understanding and addressing vicarious trauma. Headington Institute.

Perry, B. D. (2014). The cost of caring: Understanding and preventing secondary traumatic stress when working with traumatized and maltreated children. CTA Parent and Caregiver Education Series, 2(7), Child Trauma Academy Press, $1-5$.

Reese, R., \& Loanne, R. (2012). Deep mentoring: Guiding others on their leadership journey. Downers Grove, IL: InterVarsity Press.

Reynolds, V. (2011). Resisting burnout with justice-doing. International Journal of Narrative Therapy and Community Work, 4, 27-45.

Saakvitne, K. W., \& Pearlman, L. A. (1996). Transforming the pain: A workbook on vicarious traumatization. New York, NY: W.W. Norton. 
SAMHSA. (2014). Substance Abuse and Mental Health Services Administration. Trauma-Informed Care in Behavioral Health Services. Treatment Improvement Protocol (TIP) Series 57, HHS Publication No. (SMA) 13-4801. Rockville, MD: Substance Abuse and Mental Health Services Administration, 2014.

Scazzero, P. (2006). Emotionally healthy spirituality. Nashville, TN: Nelson.

Sharp, C., \& Ligenza, L. (2012). Is your organization trauma-informed? National Council for Community Behavioral Healthcare.

Simmons, B., Goot, J., Nelson, D., \& Little, M. (2009). Secure attachment: Implications for hope, trust, burnout, and performance. Journal of Organizational Behavior, 30, 233-247.

Skinner, N., \& Roche, A. (2005). Stress and burnout. A Prevention Handbook for the Alcohol and Other Drugs Workforce. National Centre for Education and Training on Addiction (NCETA). Australia: Flinders University.

Stamm, H. B. (2009). Professional Quality of Life Scale Manual (2nd Edition). Potatello, ID: ProQOL.org.

Terr, L. C. (1991). Childhood traumas: An outline and overview. American Journal of Psychiatry, 1(48), 10-20.

Trauma-informed organizational self-assessment for child abuse prevention agencies (CASA). (n.d.). Retrieved from http://www.wcasa.org/file_open.php?id=800

van Dernoot Lipsky, L., \& Burk, C. (2009). Trauma stewardship: An everyday guide to caring for self while caring for others. San Francisco, CA: Berrett-Koehler Publishers.

Volk, K., Guarino, K., Grandin, M., \& Clervil, R. (2008). What about you? A workbook for those who work with others. Waltham, MA: National Center on Family Homelessness.

Workforce Development NCETA. (2005). The National Centre for Education and Training on Addiction. Retrieved from http://nceta.flinders.edu.au/ files/2912/4710/5741/WFD_TIPS_Overview.pdf 